\title{
Forensic Expertise Based on Findings through Postmortem Mammography Imaging
}

\author{
Sara Sablone ${ }^{1, *}$, Roberto Maselli ${ }^{1}$, Alessia Leggio ${ }^{1}$, Mariagrazia Calvano ${ }^{1}$, Mirko Leonardelli ${ }^{1}$, \\ Francesco Carravetta ${ }^{1}{ }^{\circledR}$, Gerardo Cazzato ${ }^{2}{ }^{\circledR}$, Antonio De Donno ${ }^{1}$, Vincenzo Lattanzio ${ }^{1}$ and \\ Francesco Introna ${ }^{1}$ \\ 1 Section of Legal Medicine, Interdisciplinary Department of Medicine, Bari Policlinico Hospital, \\ University of Bari, 70124 Bari, Italy; maselli-roberto@libero.it (R.M.); alessialeggio@hotmail.it (A.L.); \\ mg.calvano@libero.it (M.C.); mirkoleonardelli@gmail.com (M.L.); francesco.carravetta1@gmail.com (F.C.); \\ antonio.dedonno@uniba.it (A.D.D.); lattanziov@unitin.it (V.L.); francesco.introna@uniba.it (F.I.) \\ 2 Section of Pathology, Department of Emergency and Organ Transplantation, Bari Policlinico Hospital, \\ University of Bari, 70124 Bari, Italy; gerycazzato@hotmail.it \\ * Correspondence: sara.sablone@policlinico.ba.it; Tel.: +39-338-9057-823
}

Academic Editor: Eva Scheurer

Received: 12 May 2021; Accepted: 9 June 2021; Published: 12 June 2021

\begin{abstract}
In forensic cases where standard macroscopic examination and conventional radiological analysis are inconclusive, mammography can be a useful technique. This applies especially when bodies are found in an advanced state of decomposition, with the dissolution of the soft tissues, and with fragile skeletal structures. This study proposes the use of soft X-rays (i.e., mammography) in particular cases for forensic evaluation, with specific regard to the cause of death, age estimation, and body identification. Here we report three cases in which mammography was particularly useful to obtain fundamental medico-legal information. The first case involved a body that had reached the mummification stage, the second a woman who died from asphyxiation by strangulation, and the last a charred femur found in a field. Mammography uses low-energy X-rays. This technique provides increased image quality through better contrast and higher definition. In the first case, it highlighted a complete fracture of the thyroid cartilage; in the second, it revealed a complete fracture of the horn of the hyoid bone; in the last, it showed rarefaction of the reticular substance of the charred bone. As shown in this study, in selected cases, mammography is a useful tool for post-mortem imaging.
\end{abstract}

Keywords: hyoid bone; larynx; mammography; forensic pathology; soft X-rays; femur; forensic anthropology

\section{Introduction}

In the field of forensic pathology, one of the most important issues is differential diagnosis of anatomic anomalies, traumatic lesions, and lesions that have resulted from natural causes [1]. Correct diagnosis can be reached through macroscopic observations of bones and cartilaginous structures, and histological studies [2]. However, forensic investigations can be particularly difficult in cases of bodies in an advanced state of decomposition [3], where there is a degradation of the soft tissues with fragile bone and cartilaginous structures, as well as for carbonized bodies.

In all these cases, a multidisciplinary forensic approach can be very useful to reach an accurate and precise diagnosis, which can include forensic radiological investigations [4,5]. Furthermore, if antemortem records are available for comparisons, radiology represents a useful technique for identification $[6,7]$.

Nowadays, images can be acquired using an array of modalities and techniques [8-10]: X-rays, fluorescent imaging, nuclear medicine, ultrasound imaging, tomography, and magnetic resonance 
imaging, among others. Mammography, in particular, can be used to recognize a wide range of anatomic anomalies and traumatic lesions that cannot be adequately detected through direct inspection, microdissection, or conventional radiographic study of the corpse [2,11]. Moreover, these 'soft rays' are the ideal means to study bones with incomplete ossification (e.g., the hyoid bone in young people), because the low-energy $X$-rays allow better evaluation of soft tissue and structures with the low calcium content. As the study of the growth plates and bone fusion represents a valid predictor of age and sex, mammography can also provide invaluable support in forensic anthropology [12].

The use of mammography in post-mortem imaging is poorly documented and probably underestimated in its real diagnostic potential. Indeed, only a few studies are available in the scientific literature on the advantageous application of radiographic magnification by dedicated mammographic equipment of the hyoid-larynx complex [2,3], which has been more frequently examined by conventional X-rays and CT-scan [11].

The radiographic investigations carried out in the three forensic cases here presented were performed by using a new-generation mammographer, which proved to be essential for the detection of important elements and the solution of specific medico-legal questions.

\section{Materials and Methods}

This study is based on a collection of three cases examined over 15 years, in which soft X-ray analyses of body parts were particularly useful to reach the correct forensic interpretation. The autopsies were performed at the Institute of Legal Medicine of the University of Bari. A selected body part of each corpse was analyzed by mammography: a larynx, a hyoid bone, and a femur. The body parts were examined with dedicated mammographic equipment. The images were acquired using the "digital standard" and "thermosynthesis" modes with a digital mammographer (Hologic, Inc. Marlborough, MA, USA), characterized by appropriately collimated radiant beam and $0.1 \mathrm{~mm}$ microfocal spot, useful for direct magnification of image with a 1.5-2X magnification ratio at a focus-film distance of $60-65 \mathrm{~cm}$. Exposure was set automatically by the mammographer $(23 \mathrm{kV}, 45 \mathrm{~mA})$ based on the thickness of the anatomical specimen. Then, the same images were developed using the C-VIEW software, to emphasize the contrast. Orthogonal and oblique scans were taken with this tool, as necessary. For observation of the neck structures, such as the larynx complex and the hyoid bone, a period of 20 days of fixation in $20 \%$ formalin was necessary. In cases \#1 and \#3, digital elaborations of images were performed, while in case \#2 images were directly examined from the mammography.

\subsection{Case \#1}

In May 2019, the autopsy of a 33-year-old man who had died 4 years previously was performed. In 2015, the case was dismissed as suicide by hanging. However, in 2019, the case was revisited, and it was necessary to determine the actual cause of death by exhumation and autopsy. The corpse had reached the mummification stage. On the upper anterior skin surface of the neck, there was a hyperchromic and ribbon-like area $(4 \times 1.2 \mathrm{~cm})$ that was transversally orientated. Despite a long time from death, an accurate external examination and the autopsy ruled out the presence of other significant lesions in the corpse. To examine the hyoid-larynx complex, it was excised together with the tongue by using Ghon's technique (en bloc). Then, without exerting any traction, the remnants of the pharynx and the esophagus were dissected from the dorsal wall of the hyoid-larynx complex. In this way, we avoided overlooking important laryngeal injuries as well as destroying them [13]. The macroscopic examination of the structures failed to reveal certain signs of violence, even if the superior horns of the thyroid cartilage showed unusual hypermobility, suggesting local fractures (Figure 1a,b). 


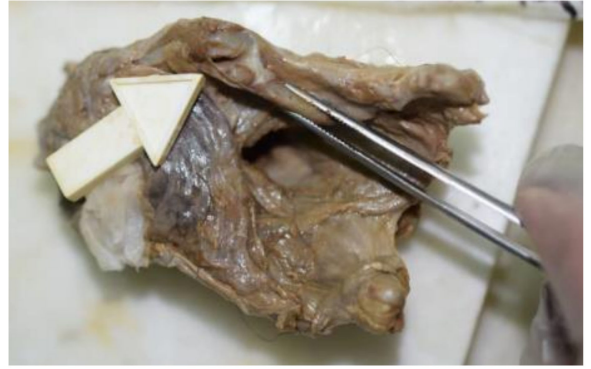

(a)

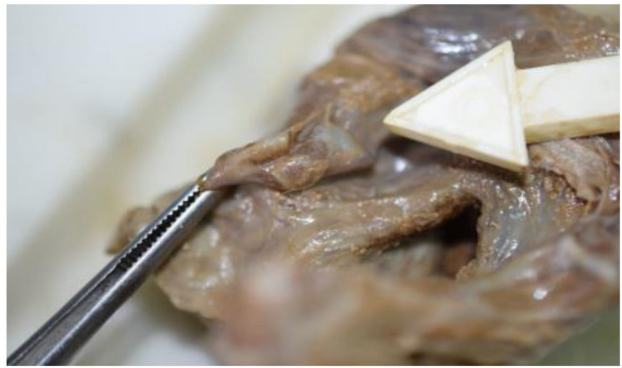

(b)

Figure 1. $(\mathbf{a}, \mathbf{b})$ The superior horns of the thyroid cartilage, apparently fractured on macroscopic examination.

Therefore, the hyoid-larynx complex was fixed in formalin to prepare it for subsequent mammographic analysis.

\subsection{Case \#2}

In 2004, the body of a 21-year-old woman was found in an abandoned field. It was bound with two pieces of rope, one wrapped four times around the neck, and the other used to tie the upper and lower limbs. The corpse had reached a stage of chromatic-emphysematous putrefaction. After external examination and the histological study, asphyxiation by strangulation was identified as the cause of death. For this reason, an in-depth study of the neck structures was performed to verify the presence of any post-traumatic lesions of the hyoid-larynx complex.

\subsection{Case \#3}

In July 2019, charred human bone remains were found in an abandoned field. The identity of the deceased and the cause and time of death were not known. It was not possible to detect lesions that might have resulted from violent or traumatic actions due to the complete carbonization of the bones. However, from among these bones, the two femurs were studied, as they are usually more resistant to environmental factors and can thus carry more information for body identification (Figure 2) [14]. The right femur had a head femur replacement. The left femur was ash-colored and with 'heat fractures' all over its structure. Despite the presence of this severe state of deterioration, it has been subjected to an anthropological and radiological examination to infer information useful for identification purposes.

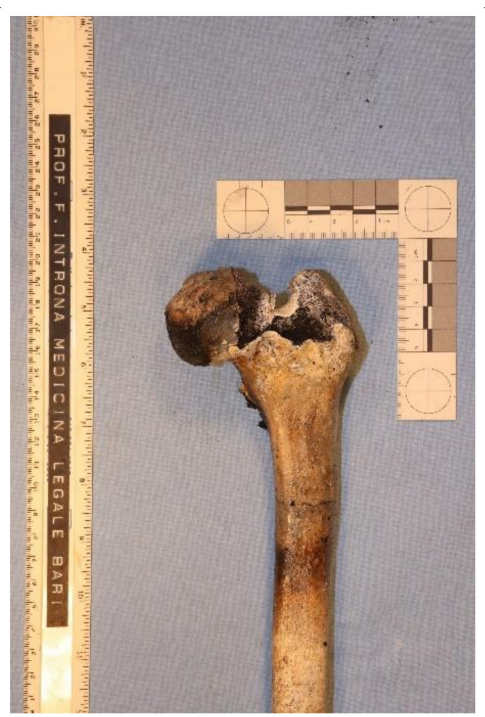

Figure 2. Charred left femur. 


\section{Results}

\subsection{Case \#1}

When mammography of the fixed larynx was performed, the suspicion of fractures of the superior horns of the thyroid cartilage was confirmed (Figure 3). The fractures were bilaterally completed-tripartite for the right horn and bipartite for the left one. The hyoid bone was whole.

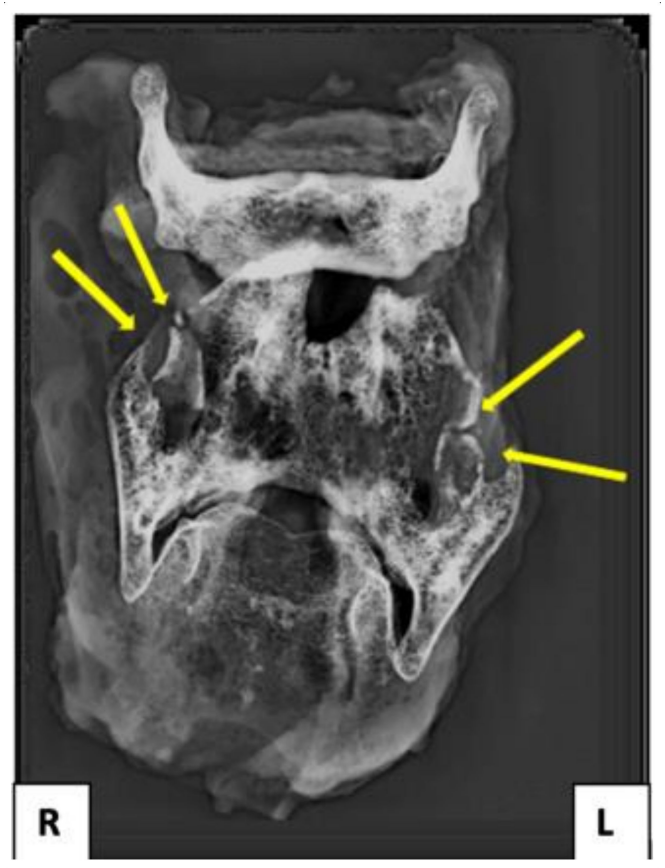

Figure 3. Bilateral fracture of the superior horns of the thyroid cartilage, examined in anteroposterior view. The arrows on the right side point the tripartite fracture and the ones on the left side the bipartite fracture (R: right; L: left).

\subsection{Case \#2}

The macroscopic observation of the hyoid-larynx complex showed incomplete ossification of the hyoid bone because of the victim young age. Indeed, the ossification process for the hyoid bone starts around the third decade of life and can be completed within a variable time (even up to 75-95 years) $[14,15]$. The mammography that was then carried out showed a complete fracture of the right horn of the hyoid bone (Figure 4).

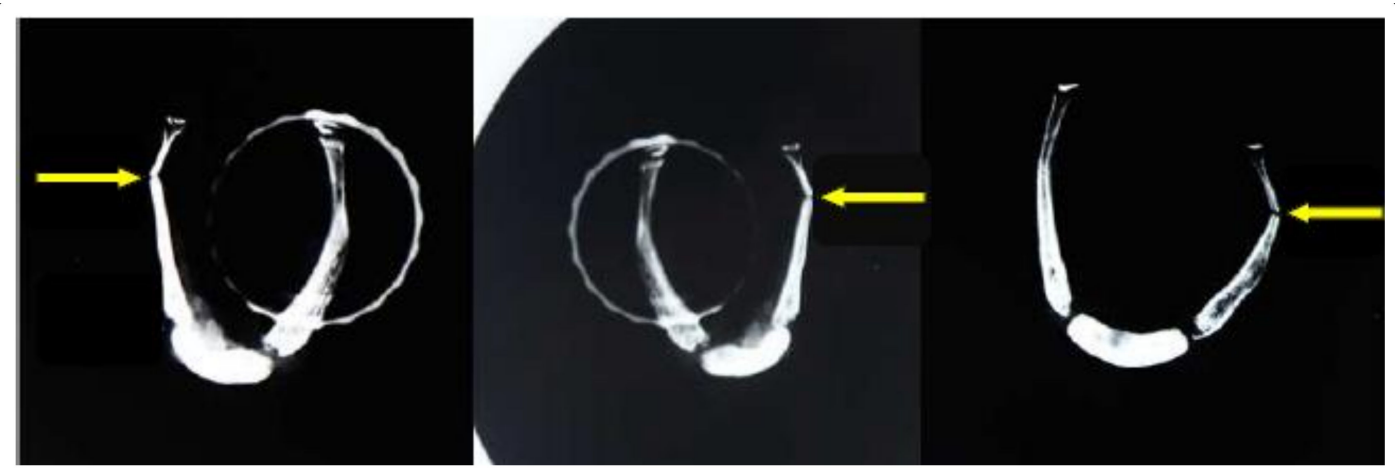

Figure 4. Complete fracture of the right horn of the hyoid bone (arrows), examined in horizontal view. 


\subsection{Case\#3}

The mammography of the left femur demonstrated rarefaction of the bone reticular substance (Figure 5), absence of conjugation cartilage residues, heat fracture residues, and a bone callus of the femoral neck, due to a non-recent trauma.

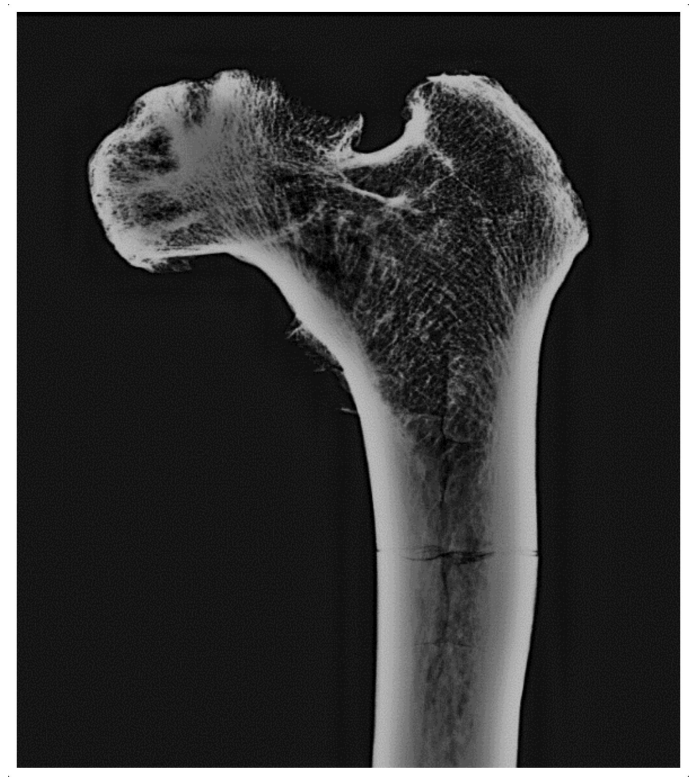

Figure 5. Rarefaction of the reticular substance of the left femur head, examined in anteroposterior view.

\section{Discussion}

Imaging techniques are of increasing importance in post-mortem investigations, especially in forensic and legal medicine [8], where their use is nearly as old as radiology itself [9]. Today's most frequently used techniques include conventional radiography, postmortem computed tomography (PMCT), post-mortem magnetic resonance imaging (PMMR), post-mortem computed tomographic angiography (PMCTA), and 3D surface documentation (or photogrammetry) [10]. However, the indications for using each method are different, due to their specific advantages and limitations [5]. For example, conventional radiology, as well as PMCT, are useful for visualization of the skeletal system and detection of foreign bodies, but they are very limited for examination of soft tissues, organs, and vascular systems. Conversely, PMMR ensures an excellent soft-tissue contrast without the use of radiation, but it is more difficult to handle, more expensive, time-consuming, and needs training for correct interpretation $[5,10]$.

Mammography is a radiologic technique usually used to examine the human breast. It employs lower-energy X-rays (i.e., usually around $30 \mathrm{kVp}$ ) than those necessary for conventional radiography of bones. Its diagnostic potential in post-mortem imaging is scarcely documented and probably underestimated since only a few studies on mammography forensic applications are available [2,3]. Indeed, through the magnification, a mammographer with low energy radiation ensures visualization of small and thin objects with low radio-opacity and can provide good quality images, with an increase in contrast and definition, and noise reduction [3].

Undoubtedly, the choice of this radiological technique must be correlated to the anatomical specimen and the state of corpse conservation [3]. For example, the larynx, as well as the bone trabecular substance (especially if rarefied), have a relatively low radiographic contrast, which may be enhanced by examining them by a mammographer. On the contrary, a conventional radiography examination on these anatomical structures would not detect any crucial evidence to reach a correct diagnosis. 
The usefulness of mammography on the hyoid-larynx complex is even more evident in cases of decomposed or putrefactive specimens, where it is generally impossible to detect signs of macroscopic hemorrhagic infiltration or other traces suggesting that the lesion was caused by an external force $[3,16]$.

Mammography is very easy and fast to perform (no more than 10-15 min are necessary between the taking of the $\mathrm{X}$-ray and reading the images) and non-expensive. Moreover, although it can acquire only two-dimensional images (as in the case of conventional radiography), adequate preparation by cautious dissection of the anatomical complex allows avoiding other organ image superimposition.

\subsection{Case \#1}

The larynx complex consists of several cartilaginous and muscular tissues. There are six cartilages, as three unpaired (the thyroid cartilage, cricoid cartilage, epiglottis) and three paired (arytenoid cartilage, corniculate cartilage, and cuneiform cartilage), together forming the mammalian larynx skeleton. The thyroid cartilage, in particular, has two projections upwards and downwards: the superior horn (cornu) and the inferior horn.

Fractures of the superior horn are common findings in suicidal hanging, because of the weakness of their internal structure. This evidence is more frequently related to older people and incomplete hanging [17].

Nevertheless, this kind of lesion is often hard to detect during a normal macroscopic examination of the larynx structures, especially when the corpse is in an advanced state of decomposition or in subjects dying immediately after sudden neck compression [18]. In fact, in these latter cases, traumas of the neck structures may not produce evident hemorrhagic infiltrations. Conversely, ambiguous macroscopic aspects, with hemorrhage of the neck soft tissues suggesting traumas, may be found in asphyxia preceded by convulsions and agony, even in the absence of neck fractures [2].

In all these cases, radiographic evaluations of specimens are crucial. In case \#1, in particular, the in-depth study of the throat skeleton to detect fractures was performed by using mammography.

This diagnostic tool was chosen because the thyroid cartilage is a soft tissue containing low levels of calcium. It is not radiopaque, so the radiographic contrast is relatively low, and not well evaluable with conventional radiography. This problem has been solved by using low-energy X-rays produced by the mammographer. Through this technique, we were able to increase the quality, contrast, and definition of the images, and thus highlight any traumatic larynx lesions.

Moreover, because the body was mummified, the larynx soft tissues were retracted and scarcely handleable. Thus, mammography allowed us to even overcome the diagnostic limits in macroscopic analysis deriving from post-mortem transformative processes. Mummification could also explain the reduction of the space between hyoid bone and larynx highlighted at the mammography (in contrast to the extension of this space in the hanged people) [2], most probably secondary to the massive and rapid tissue dehydration and retraction.

\subsection{Case \#2}

The hyoid bone has a central part, known as the body, and two pairs of horns, which are classified, on each side (right and left) as greater and lesser ones. Typically, the fusion of the body with the horns occurs in adulthood, starting at the age of about 25 years [12,14]. Only growth plates at different stages of ossification can be detected before then. The growth plate is the hyaline cartilage localized in the part of the bone where new bone growth takes place.

In the forensic field, the radiographic study of the hyoid bone is useful in case of death by manual strangulation, due to its susceptibility to fracture [11]. This kind of lesion is related both to the cause of death and to the age of the victim, being more frequent in older victims than in young ones. This can be explained by the resilience of the hyoid bone in persons under 20 years and the high flexibility of their unossified laryngeal cartilages, which allow reversible deformation under mechanical effects without the emergence of fractures [19,20]. 
Case \#2 involved a young woman (age 21) who was killed by manual strangulation. Despite the young age of the victim, during the autopsy examination, it was possible to assume that the hyoid bone had been fractured. In this case, the use of standard X-rays would not have been useful, because of the incomplete fusion and ossification of the body and horns of the hyoid bone as well as because of the partial post-mortem tissue decomposition. Moreover, the victim was female, which is the gender that shows in adulthood a significantly lower bone density than men of the same age, according to Fisher et al. [12].

Although various radiological tools, such as CT-scans, have been usefully applied in in-vivo studies of hyoid bone fusion and bone density across the lifespan [12], to the best of our knowledge there are no systematic radiological studies in the scientific literature on post-mortem hyoid bone density loss and laryngeal alterations due to cadaveric decomposition and environmental action on the corpse. Undoubtedly, factors such as local climate, soil composition, soil $\mathrm{pH}$, and vegetation cover, as well as the type and growth of microflora both in the environment and in the corpse are responsible for some post-mortem microstructural changes of the skeleton and cartilages [21,22].

For all these reasons, mammography proved to be the most useful radiological tool for identifying any hyoid lesions allowing observation of the complete fracture of the right horn of the hyoid bone. This is because of the low energy of $X$-rays used, which can penetrate the cortex and reach the core of the bone without being reflected by the cortical layer.

Mammography also contributed to establishing that the hyoid bone showed bilateral non-fusion between the body and the cornua, thus providing useful anthropological data for identification purposes and ruling out the risk of lesion overestimation.

\subsection{Case \#3}

In forensic anthropology investigations, it is possible to date skeletal remains by using the Acsàdi and Nemeskéri femur scale (Figure 6) [23,24]. It allows for establishing the skeletal age according to the characteristics and aspects of the bone reticular substance.

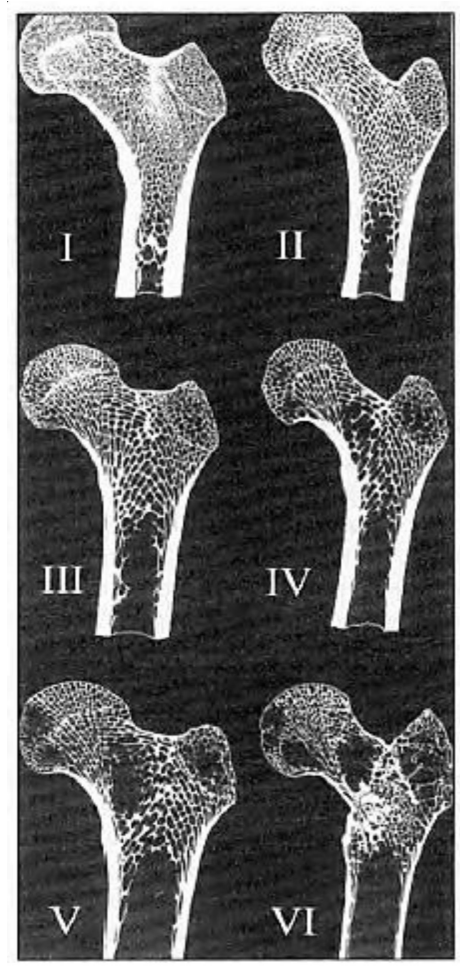

Figure 6. Iconographic representation of the six stages of the Acsàdi and Nemeskéri femur scale. 
According to this scale, the structural modifications of the femur head in terms of skeletal age can be evaluated based on:

- $\quad$ position of the apex of the medullary cavity;

- structure, modification, and rarefaction of the trabeculae.

Based on the different associations of these two indices, Acsàdi and Nemeskéri defined six different phases of morpho-structural changes of the proximal epiphysis of the femur (Table 1).

Table 1. The Acsàdi and Nemeskéri combined system for the six different phases of morpho-structural changes of the proximal epiphysis of the femur.

\begin{tabular}{ccccc}
\hline Morphological & Mean Age & Standard & \multicolumn{2}{c}{ Age Range (Years) } \\
\hline Phase & (Years) & Deviation (Years) & Actual & Calculated (3 $\times$ SD) \\
\hline I & 31.4 & - & $18-52$ & $36.2-51.8$ \\
\hline II & 44.0 & 2.60 & $19-61$ & $47.0-58.2$ \\
\hline III & 52.6 & 1.87 & $23-72$ & $49.0-63.0$ \\
\hline IV & 56.0 & 2.32 & $32-86$ & $56.8-69.9$ \\
\hline V & 63.3 & 2.17 & $38-84$ & $56.9-78.7$ \\
\hline VI & 67.8 & 3.64 & $25-85$ & - \\
\hline
\end{tabular}

Under standard conditions, the above-mentioned anthropological analysis shall be applied by carrying out a longitudinal section, on the frontal plane, of the proximal femur, to macroscopically observe the trabecular substance. However, in the case of bone carbonization and/or considerable demineralization, as in the third one here presented, it is not possible to dissect the femur because of its fragility and of the related risk of irreparable damaging. In such conditions, it is mandatory to find an alternative tool to observe the trabecular substance by preserving the bone structure [14].

Therefore, a non-invasive radiographic tool as mammography was applied. The use of low-energy X-rays allowed to penetrate the bone and to avoid cortical reflection, unlike standard radiography. In this way, it has been possible to analyze the trabecular substance. Moreover, the acquired frontal-view bidimensional images of the femur head were ideal for the comparative examination with the reference images of the Acsàdi and Nemeskéri femur scale. The comparison between the mammography scans and the Acsàdi and Nemeskéri combined system allowed us to establish that the age of the human remains was $60.0 \pm 13.2$ years. These data, together with the detection of a femoral neck bone callus from non-recent trauma and the circumstantial elements, has been of remarkable aid in the victim identification procedures.

\section{Conclusions}

In conclusion, we believe that despite the introduction of more advanced post-mortem imaging techniques, mammography can be considered a very helpful method of analysis in forensic investigations for its diagnostic accuracy and highly informative potential. Undoubtedly, this imaging technique has to be chosen according to the particulars of a case, to increase the sensitivity of the post-mortem exam. Unlike the standard radiographic methods and the independent use of macroscopic inspection, mammography has proven to be decisive for the diagnosis of the cause of death and age-at-death estimation in cases with decomposed bodies, or with altered soft tissues, or with carbonized remains and fragile bone and cartilaginous structures. Mammography also shows several advantages: it is non-invasive, reliable, readily available, inexpensive, easy to perform, and applicable to different parts of the body. Furthermore, it is not operator-dependent.

However, it is important to emphasize that this diagnostic tool must be used in combination with the more conventional post-mortem procedures, within a multidisciplinary approach. Indeed, 
mammography, as well as for other post-mortem imaging techniques, cannot replace traditional autopsy nor histopathology, or immunohistochemistry, the only ones able of establishing the viability of any bone or cartilage lesions radiographically identified.

Finally, forensic expertise based on findings through post-mortem imaging cannot disregard training in the correct interpretation of images.

The diagnostic application of mammography on other anatomical structures and in other medico-legal questions is the subject of an ongoing preliminary study by the Authors of the present report.

Author Contributions: Conceptualization, S.S. and F.I.; methodology, S.S. and F.I.; software, V.L. and R.M.; validation, F.I. and S.S.; formal analysis, R.M., F.C., and G.C.; investigation, S.S. and R.M.; resources, F.I.; data curation, S.S. and A.L.; writing — original draft preparation, S.S. and M.C.; writing-review and editing, S.S. and M.L.; visualization, G.C.; supervision, S.S. and A.D.D.; project administration, F.I. All authors have read and agreed to the published version of the manuscript.

Funding: This research received no external funding.

Institutional Review Board Statement: Not applicable.

Informed Consent Statement: Informed consent was obtained from all subjects involved in the study.

Data Availability Statement: Not applicable.

Acknowledgments: The Authors thank Christopher Berrie, former Professor of Scientific English at the University "G. D'Annunzio" in Chieti-Pescara, for his English revision.

Conflicts of Interest: The authors declare no conflict of interest.

\section{References}

1. Brogdon, B.G.; Sorg, M.H.; Marden, K. Fingering a murderer: A successful anthropological and radiological collaboration. J. Forensic Sci. 2010, 55, 248-250. [CrossRef] [PubMed]

2. Di Nunno, N.; Lombardo, S.; Costantinides, F.; Di Nunno, C. Anomalies and alterations of the hyoid-larynx complex in forensic radiographic studies. Am. J. Forensic Med. Pathol. 2004, 25, 14-19. [CrossRef] [PubMed]

3. Di Nunno, N.; Costantinides, F.; Bernasconi, P.; Lombardo, S. Radiographic magnification in the diagnosis of traumatic lesions of the hyoid-larynx complex. Int. J. Legal Med. 1998, 111, 38-42. [CrossRef] [PubMed]

4. Khokhlov, V.D. Injuries to the hyoid bone and laryngeal cartilages: Effectiveness of different methods of medico-legal investigation. Forensic Sci. Int. 1997, 88, 173-183. [CrossRef]

5. Grabherr, S.; Baumann, P.; Minoiu, C.; Fahrni, S.; Mangin, P. Post-mortem imaging in forensic investigations: Current utility, limitations, and ongoing developments. Res. Rep. Forensic Med. Sci. 2016, 6, 25-37. [CrossRef]

6. Kumar, R.; Athota, A.; Rastogi, T.; Kumar Karumuri, S. Forensic radiology: An emerging tool in identification. J. Indian Acad. Oral Med. Radiol. 2015, 27, 416-422.

7. Santoro, V.; Mele, F.; Introna, F.; De Donno, A. Personal identification through digital photo superimposition of dental profile: A pilot study. J. Forensic Odontostomatol. 2019, 3, 21-26. [PubMed]

8. Dirnhofer, R.; Thali, M.; Vock, P. The Virtopsy Approach: 3D Optical and Radiological Scanning and Reconstruction in Forensic Medicine, 1st ed.; CRC Press: Boca Raton, FL, USA, 2009.

9. Thomas, A.M.K. Post-mortem imaging-development and historical review. In Atlas of Post-Mortem Angiography; Grabherr, S., Grimm, J., Heinemann, A., Eds.; Springer International Publishing: Berlin, Germany, 2016; pp. 3-33.

10. Grabherr, S.; Egger, C.; Vilarino, R.; Campana, L.; Jotterand, M.; Dedouit, F. Modern post-mortem imaging: An update on recent developments. Forensic Sci. Res. 2017, 2, 52-64. [CrossRef] [PubMed]

11. De Bakker, H.M.; Warmbrunn, M.V.; Van den Biggelaar, P.; Soerdjbalie-Maikoe, V.; de Bakker, B.S. Fracture patterns of the hyoid-laryinx complex after fatal trauma on the neck: Retrospective radiological postmortem analysis of 284 cases. Int. J. Legal Med. 2020, 134, 1465-1473. [CrossRef] [PubMed]

12. Fisher, E.; Austin, D.; Werner, H.M.; Chuang, Y.J.; Bersu, E.; Vorperian, H.K. Hyoid bone fusion and bone density across the lifespan: Prediction of age and sex. Forensic Sci. Med. Pathol. 2016, 12, 146-157. [CrossRef] [PubMed] 
13. Maxeiner, H. "Hidden" laryngeal injuries in homicidal strangulation: How to detect and interpret these findings. J. Forensic Sci. 1998, 43, 784-791. [CrossRef] [PubMed]

14. Walker, R.A.; Lovejoy, C.O. Radiographic changes in the clavicle and proximal femur and their use in the determination of skeletal age at death. Am. J. Phys. Anthropol. 1985, 68, 67-78. [CrossRef] [PubMed]

15. Vinoth, K.V.; Vedanayagam, T. Estimation of age of ossification of hyoid bone by radiological examination of autopsy specimen. J. Indian Acad. Forensic Med. 2017, 39, 8-10. [CrossRef]

16. De Bakker, B.S.; de Bakker, H.M.; Soerdjbalie-Maikoe, V.; Dikkers, F.G. Variants of the hyoid-larynx complex, with implications for forensic science and consequence for the diagnosis of Eagle's syndrome. Sci. Rep. 2019, 9, 15950. [CrossRef] [PubMed]

17. Charoonnate, N.; Narongchai, P.; Vongvaivet, S. Fractures of the hyoid bone and thyroid cartilage in suicidal hanging. J. Med. Assoc. Thail. 2010, 93, 1211-1216.

18. Evans, K.T.; Knight, B.; Whittaker, D.K. Forensic Radiology; Blackwell Scientific: Oxford, UK, 1981; pp. $122-210$.

19. Ubelaker, D.H. Hyoid fracture and strangulation. J. Forensic Sci. 1992, 37, 1216-1222. [CrossRef] [PubMed]

20. Khokhlov, V.D. Knitted fractures of the laryngopharynx framework as a medico-legal matter. Forensic Sci. Int. 1999, 104, 147-162. [CrossRef]

21. Bell, L.S.; Skinner, M.F.; Jones, S.J. The speed of post mortem change to the human skeleton and its significance. Forensic Sci. Int. 1996, 82, 129-140. [CrossRef]

22. Ross, A.H.; Cunningham, S.L. Time-since-death and bone weathering in a tropical environment. Forensic Sci. Int. 2011, 204, 126-133. [CrossRef] [PubMed]

23. Acsàdi, G.Y.; Nemeskéri, J. History of Human Life Span and Mortality; Balás, K., Translator; Akadémiai Kiadò: Budapest, Hungary, 1970.

24. Iscan, M.Y.; Steyn, M. Skeletal age. In The Human Skeleton in Forensic Medicine, 3rd ed.; Charles C Thomas Publisher Ltd.: Springfield, IL, USA, 2013; pp. 59-143.

Publisher's Note: MDPI stays neutral with regard to jurisdictional claims in published maps and institutional affiliations.

(C) 2021 by the authors. Licensee MDPI, Basel, Switzerland. This article is an open access article distributed under the terms and conditions of the Creative Commons Attribution (CC BY) license (http://creativecommons.org/licenses/by/4.0/). 